estimate the cost and morbidity associated with the diagnostic investigation of patients with tumefactive demyelination (TD) compared to patients with conventional relapsing-remitting MS.

Methods Retrospective review of patients seen between 20132018 in clinics at the Brain and Mind Centre, Sydney; a centre with tertiary referral expertise in MS. Records were searched for the terms 'tumefactive' and 'pseudotumour'. All patients diagnosed with TD were included and paired with a patient of similar age diagnosed in the same year with MS according to 2010 McDonald criteria.

Results There were 31 patients with TD and 31 patients with conventional relapsing remitting MS. The estimated cost of investigating TD was more than 7.5 times higher per patient than in MS $(\$ 18,300$ AUD vs $\$ 2,418$ AUD, $\mathrm{p}<0.001)$. Brain biopsy was performed in 6/31 TD patients and $0 / 31$ MS patients and was the cause of more adverse outcomes in the TD versus MS group. More patients in the TD group were admitted to hospital (22/31 vs $10 / 31)$ and ICU admissions only occurred in the TD group (10/22 vs $0 / 10)$.

Conclusion The cost and adverse outcomes associated with investigating $\mathrm{TD}$ are higher than in conventional MS. Improvements in the diagnosis of TD have the potential to improve health and economic outcomes.

\section{ACUTE CEREBELLAR ATAXIA FOLLOWING EPSTEIN-BARR VIRUS INFECTION}

${ }^{1}$ Stephanie L Barnes*, 2,3,4Bruce J Brew. 'Department of Neurology, St Vincent's Hospital, Darlinghurst, NSW, Australia; ${ }^{2}$ Departments of Neurology and Immunology, St Vincent's Hospital, Darlinghurst, NSW, Australia; ${ }^{3}$ Peter Duncan Neurosciences Research Unit, St Vincent's Centre for Applied Medical Research, Darlinghurst, NSW, Australia; ${ }^{4}$ St Vincent's Clinical School, Faculty of Medicine, University of NSW and University of Notre Dame Sydney, Sydney, NSW, Australia

\subsection{6/jnnp-2019-anzan.37}

Introduction Infectious aetiologies such as acute Epstein-Barr virus (EBV) infection are in the differential diagnosis for acute cerebellar ataxia (ACA). This syndrome remains exceptionally rare and not well characterised in adults. ${ }^{\text {e.g. }} 12$

Methods A retrospective case review of a patient diagnosed with ACA following EBV infection with implications for pathogenesis and treatment.

Results A 29-year-old Caucasian male presented with a three day history of ACA. Seven days prior he was diagnosed with infectious mononucleosis; bloodwork was consistent with acute EBV infection. These symptoms improved rapidly with oral prednisolone. He took no regular medications, drank alcohol moderately and had no significant family history.

On examination, he was afebrile, ataxic and mildly dysarthric. Sensory examination was normal, particularly proprioception. Romberg's test was negative. Remaining neurological and general examination was normal.

Bloodwork showed mild liver dysfunction and positive ANA (titre 1/320, homogenous and speckled patterns). Immune screen was otherwise negative. Antineuronal antibody panel was negative in serum and CSF. CSF glucose was 3.1 $\mathrm{mmol} / \mathrm{L}$, protein $751 \mathrm{mg} / \mathrm{L}$, albumin $523 \mathrm{mg} / \mathrm{L}$, neopterin 24 $\mathrm{nmol} / \mathrm{L}$ and $\mathrm{B}_{2}$ microglobulin $1.1 \mathrm{mg} / \mathrm{L}$. The sample was acellular with negative EBV PCR ( $<500$ copies $/ \mathrm{mL})$. Other infective serology and PCRs were also negative. MRI brain with gadolinium showed no abnormality.

The patient received supportive care and was neurologically normal within three months.

Conclusions ACA related to EBV is rare in adults. This report is important because it documents an adult case, other ACA causes have been rigorously excluded, resolution without antiviral therapy is detailed, and investigations support an immune-mediated pathogenesis.

\section{REFERENCES}

1. $\mathrm{McC}$ arthy $\mathrm{CL}, \mathrm{McC}$ lgan $\mathrm{P}$, Martin P. Acute cerebellar ataxia due to Epstein-Barr virus. Pract Neurol 2012;12:238-240.

2. Lascelles RG, Longson M, Johnson PJ, Chiang A. Infectious mononucleosis presenting as acute cerebellar syndrome. Lancet 1973;2:707.

\section{RESPIRATORY FUNCTION AND COGNITIVE PROFILE IN MOTOR NEURON DISEASE}

${ }^{1}$ William Huynh*, ${ }^{2}$ Lara E Sharplin, 'Jashelle Caga, 'Elizabeth Highton-Williamson, ${ }^{1}$ Matthew C Kiernan. 'Brain and Mind Centre, Camperdown, NSW, Australia; ${ }^{2}$ Faculty of Medicine, The University of Notre Dame, Sydney, NSW, Australia

\subsection{6/jnnp-2019-anzan.38}

Introduction Motor neuron disease (MND) is increasingly recognised as a multisystems disorder with $30-50 \%$ of patients having mild to moderate cognitive impairment. Mechanisms of cognitive dysfunction in MND are multifactorial but chronic hypoxia secondary to respiratory dysfunction may contribute to cognitive decline in patients.

Objectives The current study aimed to identify the relationship between respiratory function in MND patients and the presence and degree of cognitive impairment.

Methods MND patients were prospectively recruited from a multidisciplinary MND clinic. Patients meeting the criteria for frontotemporal dementia were excluded. Baseline clinical assessments including respiratory function as assessed by spirometry were recorded with FVC $\leq 75 \%$ considered to have reduced respiratory function. Cognitive testing was performed utilising the Addenbrooke's Cognitive Examination (ACE).

Results From a cohort of 100 MND patients 48\% were categorised as having impaired respiratory function whilst 52\% had normal function. Compared to the group with normal respiratory function (ACE: $86.83 \pm 1.5$ ), patients with respiratory dysfunction had significantly reduced cognitive function (ACE: 90.68 $\pm 0.89, \mathrm{P}=0.025$ ). Subscores demonstrated significant differences between the groups with respect to domains in memory, attention with a trend observed in fluency. There was a significant correlation between FVC and ACE scores as well as between FVC and memory and fluency subscores $(\mathrm{P}<0.01)$.

Conclusion MND patients with respiratory compromise were more likely to develop reduced cognitive function. In addition to improving physical function, it remains plausible that noninvasive ventilation may alter the progression of cognitive impairment in MND patients, thereby potentially improving their overall quality of life and carer burden. 